\title{
Mutual geometry of confocal Keplerian orbits: uncertainty of the MOID and search for virtual PHAs
}

\author{
Giovanni F. Gronchi, Giacomo Tommei and Andrea Milani \\ Department of Mathematics, University of Pisa, Largo B. Pontecorvo 5, 56127 Pisa, Italy \\ email: gronchi@dm.unipi.it
}

\begin{abstract}
The Minimum Orbit Intersection Distance (MOID) between two confocal Keplerian orbits is a useful tool to know if two celestial bodies can collide or undergo a very close approach. We describe some results and open problems on the number of local minimum points of the distance between two points on the two orbits and the position of such points with respect to the mutual nodes. The errors affecting the observations of an asteroid result in uncertainty in its orbit determination and, consequently, uncertainty in the MOID. The latter is always positive and is not regular where it vanishes; this prevents us from considering it as a Gaussian random variable, and from computing its covariance by standard tools. In a recent work we have introduced a regularization of the maps giving the local minimum values of the distance between two orbits. It uses a signed value of the distance, with the sign given to the MOID according to a simple orientation property. The uncertainty of the regularized MOID has been computed for a large database of orbits. In this way we have searched for Virtual PHAs, i.e. asteroids which can belong to the category of PHAs (Potentially Hazardous Asteroids) if the errors in the orbit determination are taken into account. Among the Virtual PHAs we have found objects that are not even NEA, according to their nominal orbit.
\end{abstract}

Keywords. MOID, Potentially Hazardous Asteroids, orbit determination

\section{Introduction}

The orbit distance between two Keplerian orbits with a common focus is useful to know if two celestial bodies moving along these orbits can collide or undergo a very close approach. If the orbit distance is large enough there is no possibility of such an event, at least during the time span in which the Keplerian solutions are a good approximation of the real orbits. This distance is called MOID (Minimum Orbit Intersection Distance) in the literature; this acronym was introduced in (Bowell \& Muinonen 1994) with the definition of Potentially Hazardous Asteroids (PHA), an asteroid with MOID $\leqslant 0.05$ AU and absolute magnitude $H \leqslant 22$.

Two confocal Keplerian orbits can get close at more than a pair of points, for example near both the mutual nodes, thus it is useful to compute all the local minima of the Keplerian distance function d, distance between two points on the two orbits as a function of the two anomalies along the orbits (see Figure 1), not only the absolute minimum. We compute these values as the stationary points of the function $d^{2}$, squared to be smooth also in case of orbit crossing, when the distance can be zero (see Section 2).

When a new celestial body is detected and its observations are enough, the orbit of the body can be determined by means of a least squares fit. Moreover the uncertainty in the determination of the nominal orbit produced by the errors in the observations can be represented by a covariance matrix (see, e.g., Milani 1999). The errors in the orbit determination also affect the computation of the MOID, and it is important to estimate 


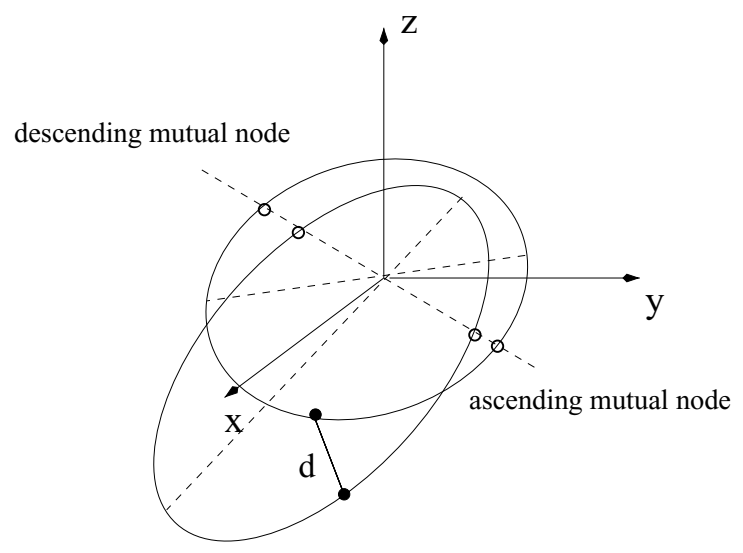

Figure 1. Two confocal orbits and the distance $d$ for two selected values of the anomalies. The apsidal lines and the mutual nodal lines (intersection of the two orbital planes) are also drawn.

the resulting uncertainty. In other cases, the covariance of some function of the orbital elements can be computed by a covariance propagation formula (see Section 3), but in the case of the MOID the possibility of orbit crossings produces a singularity in this computation. An additional difficulty is that the uncertainty of a non-zero but small orbit distance may allow negative values of the distance, that are meaningless. Both these problems prevent us from computing a meaningful confidence interval just when the MOID can be small or vanishing.

We use the results of (Gronchi \& Tommei 2007), in which we regularize the local minima of $d$ as maps of the orbit configurations according to an intuitive geometric rule. Using these regularized maps we can compute a meaningful confidence interval for the local minimal distance maps also when they vanish (see Section 4). With this new algorithm we compute the covariance of the regularized maps for a large database of orbits with covariance matrix, and we search for Virtual PHAs, i.e. asteroids which can belong to the PHA category if the errors in the orbit determination are taken into account (see Section 5). Among the Virtual PHAs we have found objects whose nominal orbits are not even Near Earth Asteroids (NEA), that is have a nominal perihelion $q>1.3$ AU.

A mathematical theory to compute the uncertainty of the orbit distance is relevant to applications such as to produce an observation priority list for follow up of NEAs based on the possible orbit distance with the Earth. We briefly comment on this in Section 6 .

\section{Stationary points of the squared Keplerian distance function}

\subsection{Computation of the stationary points of $d^{2}$}

There are several papers in the literature on the computation of the minimum points of $d$ (e.g. Sitarski 1968; Hoots 1984; Dybczynski et al. 1986). Recently some algebraic methods to compute all the stationary points of $d^{2}$ have been introduced, using the Gröbner bases (Kholshevnikov \& Vassiliev 1999) and the resultant theory (Gronchi 2002; Gronchi 2005). They are both based on a polynomial formulation of the problem. The resultant method is used to compute the MOID and all the stationary points of $d^{2}$ (also for cometary orbits) by the orbit determination software Orbfit $\dagger$ and in the NEODyS websitef. In

$\dagger$ A free software, released under a GNU Public License software, by the Orbfit consortium lead by the University of Pisa. To download see http://newton.dm.unipi.it/orbfit

$\ddagger$ The Near Earth Objects Dynamic Site, maintained at the Universities of Pisa and Valladolid, http://newton.dm.unipi.it/neodys and http://unicorn.eis.uva.es/neodys 
Table 2.1 and Figure 2.1 we show the result of the computation of the stationary points for the Near Earth Asteroid $1991 \mathrm{~TB}_{2}$. The data for all the examples in this paper are from the NEODyS site for the NEOs and from JPL ephemerides for the planets.

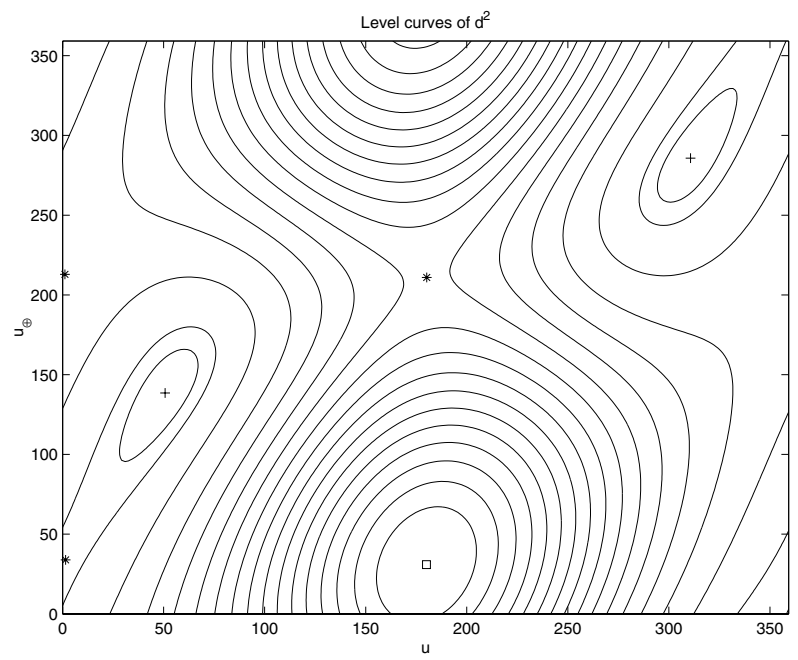

Figure 2. Level curves of $d^{2}$ for the asteroid $1991 \mathrm{~TB}_{2}$ in the plane of the eccentric anomalies $u$ (for the asteroid), $u_{\oplus}$ (for the Earth). Asterisks $\left(^{*}\right)$ are saddle points, squares ( $\square$ ) are maxima, crosses (+) are minima.

\begin{tabular}{rrrr}
\hline$u$ & $u_{\oplus}$ & $d$ & type \\
\hline 50.73 & 138.52 & 0.11 & MINIMUM \\
310.72 & 285.75 & 0.14 & MINIMUM \\
1.50 & 33.86 & 0.56 & SADDLE \\
1.15 & 212.86 & 1.45 & SADDLE \\
180.21 & 210.96 & 2.61 & SADDLE \\
180.05 & 30.90 & 4.61 & MAXIMUM \\
\hline
\end{tabular}

Table 1. Stationary points for asteroid $1991 \mathrm{~TB}_{2}$, giving the values of the eccentric anomalies (in degrees), the values of the distance $d$ (in $\mathrm{AU}$ ) and the type of stationary point.

The algebraic formulation of the problem has two main advantages: (a) allows to search for all the solutions using the efficient methods of modern Computational Algebra (Cox 1992; Bini 1997); (b) allows to give a bound for the maximum number of stationary points, as discussed below.

\subsection{Mutual geometry of confocal Keplerian orbits}

How many stationary points of $d^{2}$ may exist? And among them, how many local minima? In (Gronchi 2002) we found that the stationary points are at most 16 for the case of two ellipses and at most 12 if one orbit is circular, except for very particular cases with infinitely many stationary points. We do not have a proof that these are the optimal bounds. By a large number of numerical experiments we have found cases with at most 12 stationary points of $d^{2}$ and at most 4 local minima (see Gronchi 2002; Gronchi 2005).

In Table 2 we show a statistics of the stationary and minimum points of the distance function $d$ between points on the orbit of the Earth and on the orbit of a NEA. On August 4, 2006 the NEODyS database contained the orbits of 4102 Near Earth Asteroids. 


\begin{tabular}{llll}
\hline NEAs with 4 stationary points: & 1829 & NEAs with 1 minimum point: & 1830 \\
NEAs with 6 stationary points: & 2244 & NEAs with 2 minimum points: & 2267 \\
NEAs with 8 stationary points: & 28 & NEAs with 3 minimum points: & 5 \\
NEAs with 10 stationary points: & 1 & NEAs with 4 minimum points: & 0
\end{tabular}

Table 2. Statistics with NEODyS database. Total number of NEAs: 4102.

Note that most mutual orbit configurations from this database have 2 local minima of $d$, among 6 stationary points. This is the most intuitive case, with a simple geometry. Moreover at least one maximum point must always exist, for topological reasons ( $d$ is a continuous function defined on a compact set, more precisely a 2 -torus). There are also several cases with only 1 local minimum of $d$ : surprisingly enough, the asteroid 2000 $\mathrm{DK}_{79}$ shows 2 maximum and 1 minimum point, that is not intuitive at all. This explains the difference of one unit between the NEAs with 4 stationary points and the NEAs with only 1 minimum. From a classical mathematical theory (see Milnor 1963) there is a simple relation among the stationary points, apart from special cases, in which the Hessian matrix of $d^{2}$ evaluated at the stationary points is degenerate. Generically

\section{NUMBER of MIN. + MAX. POINTS $=$ NUMBER of SADDLE POINTS .}

The only NEA so far discovered with 10 stationary points is 2004 LG; this asteroid and $1997 \mathrm{US}_{9}, 2004 \mathrm{BU}_{58}, 2004 \mathrm{XM}_{14}, 2005 \mathrm{NK}_{1}$ have 3 minimum points. No real asteroid has been found so far with 4 minimum points, although we know this case is possible from numerical experiments conducted with large sets of fictitious orbits.

Is there a geometric method to locate the minimum points along the orbits? When there is a crossing between the orbits $(\mathrm{MOID}=0)$ the minimum point of $d$ corresponds to a mutual node. Is it always true that at least a local minimum point of $d$ is close to a mutual node? The answer is negative: we can find examples, like asteroid $1991 \mathrm{~TB}_{2}$ (see Figure 2.1 and Table 2.1), with two minima, both far enough from the mutual nodes. We can understand such cases arising from orbit configurations with low mutual inclination.

\section{Uncertainty of the MOID}

In this section we describe a method to define a meaningful uncertainty for all the local minima of $d$ (see Gronchi \& Tommei 2007), and in particular for the MOID, taking into account the uncertainty of the orbit of the asteroids. The second orbit is always the one of the Earth, so that its uncertainty is negligible. Nevertheless the method would allow to take into account also the uncertainty of the second orbit.

\subsection{Uncertainty of the orbit}

The observations of a celestial body are affected by errors, producing an error in the determination of the orbit. Let $(E, v)$ be a set of orbital elements: $E$ describes the geometric configuration of the orbit and $v$ is a parameter along the trajectory. For example we can use the 5 Keplerian elements $E=(a, e, I, \Omega, \omega)$, and the true anomaly as $v$. For cometary orbits we can use the perihelion distance $q$ in place of $a$.

Gauss' method gives us a nominal orbit $\left(E^{*}, v^{*}\right)$, solution of a least squares fit, together with its uncertainty. The uncertainty is represented by the $6 \times 6$ covariance matrix

$$
\Gamma_{(E, v)}=\left(\begin{array}{cc}
\Gamma_{E} & \vdots \\
\ldots & \Gamma_{v}
\end{array}\right)
$$


which is the inverse of the normal matrix

$$
C_{(E, v)}=\left[\frac{\partial \Xi}{\partial(E, v)}\right]^{t}\left[\frac{\partial \Xi}{\partial(E, v)}\right],
$$

where $\Xi$ is the vector of the observational residuals. Note that the $5 \times 5$ sub-matrix $\Gamma_{E}$ gives the marginal covariance of the five elements $E$, independently from the value of the sixth one $v$, and $C^{E}=\Gamma_{E}^{-1}$ is the marginal normal matrix.

One way of representing the uncertainty of the orbital elements $E$ is by means of confidence ellipsoids, an appropriate approximation whenever the least squares problem is quasi-linear and the uncertainty is moderate (see Milani 1999). By truncating the expansion of the target function (sum of squares, suitably weighed, of the residuals) to order 2 it is possible to represent the region in the orbital elements space where the target function does not increase above the minimum by more than a given value $\sigma^{2}$ as

$$
Z_{E}(\sigma)=\left\{E \mid\left(E-E^{*}\right)^{t} C^{E}\left(E-E^{*}\right) \leqslant \sigma^{2}\right\},
$$

that is, as an ellipsoid in the space of the geometric configurations $E$. If the eigenvalues of the covariance matrix $\Gamma_{E}$ are too large this representation is inaccurate, because the truncation to order 2 is a poor approximation.

\subsection{The minimal distance maps and their singularities}

Let $E$ be the vector of 5 orbital elements representing the orbit configuration of the asteroid and $v$ the anomaly, and let $\left(E_{\oplus}, v_{\oplus}\right)$ be the orbit of the Earth, supposedly known with negligible errors.

Given the two orbit configuration $\left(E, E_{\oplus}\right)$, the Keplerian distance function can be regarded as a map $V=\left(v, v_{\oplus}\right) \mapsto d(E, V)$, with $E_{\oplus}$ as fixed parameters. Moreover the number of stationary points of $V \mapsto d(E, V)$ is constant for $E$ in a neighborhood $\mathfrak{U}$ of $E^{*}$; actually this number can change only if the $2 \times 2$ Hessian matrix of the function $V \mapsto d\left(E^{*}, V\right)$ is degenerate. For each configuration $E$ in $\mathfrak{U}$ we consider the minimum points $V_{h}(E)$ and we define the maps

$$
\begin{array}{ll}
d_{h}(E)=d\left(E, V_{h}(E)\right) & \text { local minimal distance; } \\
d_{\text {min }}(E)=\min _{h} d_{h}(E) & \text { orbit distance (MOID) }
\end{array}
$$
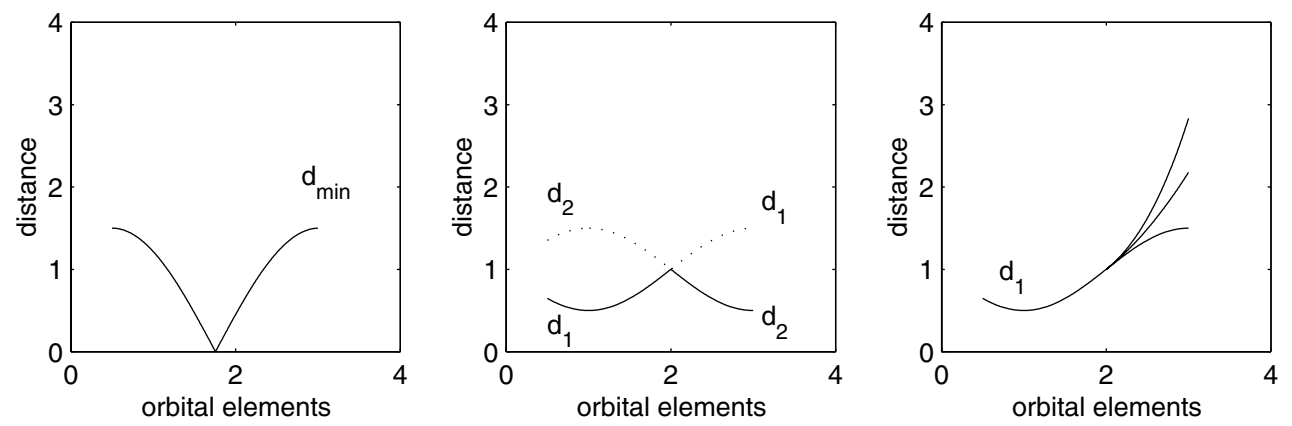

Figure 3. The singularities of the maps $d_{h}$ and $d_{\min }$ are shown in the figure: from left to write we draw a sketch of the problems described in (i), (ii), (iii).

In Figure 3 we show the singularities of $d_{h}$ and of $d_{m i n}$ :

(i) $d_{h}$ and $d_{\min }$ are not differentiable where they vanish; 
(ii) in a neighborhood of an orbit configuration $E^{*}$, two local minima can exchange their role as absolute minimum: then $d_{\min }$ can lose its regularity even without vanishing;

(iii) when a bifurcation occurs the definition of the maps $d_{h}$ may become ambiguous. Note that this ambiguity does not occur for the $d_{\text {min }}$ map. The bifurcation phenomena can occur only where the Hessian matrix of $d^{2}(E, V)$ is degenerate.

\subsection{Computation of the uncertainty of $d_{h}$ and $d_{m i n}$}

The errors in the orbit affect the computation of the local minima of $d$ : we want to estimate the size of this effect. Let us consider the orbit distance map $d_{\text {min }}$; the same method can be applied to compute the uncertainty of the minimal distance maps $d_{h}$.

For a given $\left(E^{*}, E_{\oplus}\right)$, the nominal orbit configuration $E^{*}$ being endowed with its covariance matrix $\Gamma_{E}$, we can compute the covariance of $d_{\min }\left(E^{*}\right)$ by a linear propagation of the matrix $\Gamma_{E}$ (Jazwinski 1970)

$$
\Gamma_{d_{\min }(E)}=\left[\frac{\partial d_{\min }}{\partial E}\left(E^{*}\right)\right] \Gamma_{E}\left[\frac{\partial d_{\min }}{\partial E}\left(E^{*}\right)\right]^{t} .
$$

The possibility of crossings between the orbits produces a singularity in this computation because the partial derivatives $\partial d_{\min } / \partial E\left(E^{*}\right)$ do not exist when $d_{\min }\left(E^{*}\right)=0$, e.g., when the two orbits in the configuration $\left(E^{*}, E_{\oplus}\right)$ intersect each other.

An additional problem is that the uncertainty of a non-zero but small orbit distance may allow meaningless negative values of the distance.

Note that we are interested in knowing the uncertainty just when the orbit distance can be small or vanishing, that is when a collision or a close approach is possible. Thus an algorithm exploiting the classical covariance propagation to compute the uncertainty of the MOID is available only when it is not very useful. As a result of this deeply rooted mathematical difficulty, so far all the authors discussing the distribution of the MOID of asteroid orbits (e.g., the PHA population) have given up the use of the available orbit uncertainty information, with the only exception of the paper discussed in the next subsection.

\subsection{An approximation of the MOID}

An approximation of the MOID has been proposed in Wetherill (1967) by using the straight lines tangent to the orbits at the mutual nodes and taking the distance between these lines as two approximations of the local minima. (See the comments by ReVelle on Wetherill's biography in this volume.)

In Bonanno (2000) the uncertainty for the approximated MOID is computed by using Wetherill's approximation, as a distance between two straight lines. This distance can be given a sign, in accordance with the sign of the nodal distance (positive if the asteroid and the Earth mutual nodal points and the Sun are in this order, negative otherwise). This regularizes the approximated MOID function and allows to use the covariance propagation formulae of Section 3.3.

This approach gives useful approximate results in many cases, but has the following problems:

1) if the mutual orbital inclination $I_{M}$ is zero the mutual nodes are not defined;

2) the minimum points can be located far from the mutual nodes; they also can be close to only one node;

3) the approximations of the local minima at the mutual nodes cannot be more than two while there are known cases with up to four local minimum points. 


\section{Regularization of the minimal distance maps}

In Gronchi \& Tommei (2007) a regularization of the maps $d_{h}, d_{m i n}$ is introduced. Let us take into account the map $d_{m i n}$, the same method can also be applied to $d_{h}$. It is possible to make $d_{\text {min }}$ locally analytic even where its value is zero, simply by changing its sign according to some properties of the orbit configuration.

The idea of the regularization can be illustrated by a simple example. Let us consider the positive function, defined on the whole plane, $f(x, y)=\sqrt{x^{2}+y^{2}}$ and the function $\tilde{f}$, defined on a smaller domain by $\tilde{f}(x, y)=\operatorname{sign}(x) f(x, y)$. The directional derivative of $f$ in $(x, y)=(0,0)$ does not exist for every choice of the direction. The regularized function $\tilde{f}$, extended by continuity to the origin $(0,0)$, has all the directional derivatives in $(x, y)=(0,0)$. How to extend such method to the problem at hand is discussed below.

\subsection{Geometric definition of the regularization}

Let $\tau_{1}, \tau_{2}$ be the tangent vectors to the orbits at the minimum point and let $\Delta_{\text {min }}$ be the vector joining the two tangency points $\left(\left|\Delta_{\text {min }}\right|=d_{\text {min }}\right)$. If $\Delta_{\text {min }} \neq 0$ and $\tau_{1}$ is not parallel to $\tau_{2}$ we can define the nonzero vector $\tau_{3}=\tau_{1} \times \tau_{2}$. Due to the stationary points properties $\Delta_{\min }$ is parallel to $\tau_{3}$.

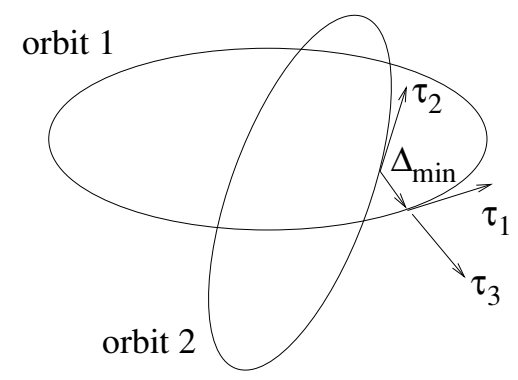

Figure 4. The orientation of the two parallel vectors $\Delta_{\min }, \tau_{3}$ is the key to define a regular map $\tilde{d}_{m i n}$ by simply changing the sign of $d_{m i n}$ on selected configurations $E$.

We define the regularized map $\tilde{d}_{\text {min }}$ by setting $\left|\tilde{d}_{\text {min }}\right|=d_{\text {min }}$ and choosing the sign + for $\tilde{d}_{m i n}$ if $\Delta_{\min }$ and $\tau_{3}$ have the same orientation, the sign - otherwise. This sign is well defined, with the only exception of the cases in which $\tau_{1}$ and $\tau_{2}$ are parallel.

Then we extend the definition domain to most crossing orbits setting $\tilde{d}_{\text {min }}=0$ if $d_{\text {min }}=0$. The orbit configurations with parallel tangent vectors to minimum points are also excluded from the definition domain even if they are not crossing points.

The resulting map $E \mapsto \tilde{d}_{\min }(E)$ is locally analytic almost everywhere (see Gronchi $\&$ Tommei 2007), including in a neighborhood of most orbit configurations $E$ such that $d_{\text {min }}(E)=0$. In particular the partial derivatives can be computed as

$$
\frac{\partial \tilde{d}_{\min }}{\partial E_{k}}\left(E^{*}\right)=\left\langle\hat{\tau}_{3}\left(E^{*}\right), \frac{\partial \Delta}{\partial E_{k}}\left(E^{*}, V_{\min }\left(E^{*}\right)\right)\right\rangle \quad k=1 \ldots 5
$$

where $V_{\min }\left(E^{*}\right)$ is the absolute minimum point and $\Delta(E, V)$ is the vector joining the points corresponding to $v$ and $v_{\oplus}$ on the orbit of the asteroid and of the Earth respectively.

Thus it becomes possible to use for the smooth function $\tilde{d}_{m i n}(E)$ the standard covariance propagation formula, applicable only to differentiable functions, including the really interesting low MOID cases. 


\subsection{Covariance of $\tilde{d}_{m i n}$}

For each configuration $\left(E^{*}, E_{\oplus}\right)$, with covariance matrix $\Gamma_{E}$, we can compute the covariance of $\tilde{d}_{\min }\left(E^{*}\right)$ as

$$
\Gamma_{\tilde{d}_{\text {min }}(E)}=\left[\frac{\partial \tilde{d}_{\text {min }}}{\partial E}\left(E^{*}\right)\right] \Gamma_{E}\left[\frac{\partial \tilde{d}_{\text {min }}}{\partial E}\left(E^{*}\right)\right]^{t}
$$

by using the smooth partial derivatives of eq. (4.1). The standard deviation, defined as

$$
\sigma_{\min }\left(E^{*}\right)=\sqrt{\Gamma_{\tilde{d}_{m i n}\left(E^{*}\right)}},
$$

allows us to define an uncertainty interval for $\tilde{d}_{\text {min }}\left(E^{*}\right)$. If we assume that the minimal distance $\tilde{d}_{h}(\overline{\mathcal{E}})$ is a Gaussian random variable, there is a high probability $(\sim 99.7 \%)$ that its value is within the interval $\left[\tilde{d}_{\min }\left(E^{*}\right)-3 \sigma_{\min }\left(E^{*}\right), \tilde{d}_{\min }\left(E^{*}\right)+3 \sigma_{\min }\left(E^{*}\right)\right]$. This statement needs to be taken with some caution. It is necessary to check that the singular case $\left(\tau_{1}\right.$ parallel to $\left.\tau_{2}\right)$ does not occur at $E^{*}$ and is not even within the confidence ellipsoid. We need to check the variance of the determinant of the Hessian matrix to look for possible bifurcations of the stationary points. Last but not least, the propagation of the covariance by the linear formula of eq. (4.2) may be mathematically consistent, but to consider $\tilde{d}_{\min }(E)$ as a Gaussian random variable is a good approximation only if the function $\tilde{d}_{\text {min }}$ is quasi-linear, when the uncertainty on $E$ is small.

In this paper we are using only this quasi-linear approximation, thus the results are meaningful only when the distance between the nominal orbit configuration $E^{*}$ and the ones corresponding to low MOID is small. This implies that the estimate of the probability is only approximate, and this approximation is useful only for comparatively high probabilities of being a PHA. Thus in the next Section we will present the results of the numerical tests on real asteroids by giving the probabilities approximated to $1 \%$ and by neglecting entirely the cases with probabilities $<1 \%$. We will also apply this method only to asteroids for which there are enough data to compute an orbit with low to moderate uncertainty.

\section{Search for Virtual NEAs and Virtual PHAs}

We have computed the uncertainty of all the local minima of $d$ using the orbit of the Earth and a large database of asteroid orbits, each with a covariance matrix representing its uncertainty. These orbits have been computed using the astrometric and photometric data made public by the Minor Planet Center (MPC) on March 2006, and they are divided into quality classes, according to their Arc-Type (see Milani et al. 2006).

The regularized distance maps $\tilde{d}_{h}, \tilde{d}_{m i n}$, and the perihelion distance $q$ can be considered as Gaussian random variables, thus for each asteroid we can decide if it is a Virtual NEA or a Virtual PHA according to the following definitions:

1) a Virtual NEA (VNEA) is an asteroid that has a nonzero probability of being a NEA, i.e. having the perihelion distance $q \leqslant 1.3 \mathrm{AU}$;

2) Virtual PHA (VPHA) is an asteroid that has a nonzero probability of being a PHA, i.e. having $\left.\operatorname{MOID}\left(=\mid \tilde{d}_{\text {min }}\right) \mid\right) \leqslant 0.05$ AU and absolute magnitude $H \leqslant 22 . \dagger$

Because of the limitations discussed above, we report only the cases in which the probabilities are $\geqslant 1 \%$. The computation of such probabilities are explained below.

$\dagger$ We are not taking into account the uncertainty of the absolute magnitude; it depends on the uncertainty in the radial geocentric distance and in a deeper analysis it should be considered. 
5.1. Probability of being a NEA

According to the standard formulae for the probability that a Gaussian random variable belongs to a given interval, we can compute the probability that for a given nominal two-orbit configuration $E^{*}$ the perihelion distance $q=q\left(E^{*}\right)$ is less than 1.3 AU as

$$
\mathcal{P}(q \leqslant 1.3 \mathrm{AU})=\frac{1}{\sqrt{2 \pi}} \int_{z_{1}}^{z_{2}} \exp \left(-z^{2} / 2\right) d z
$$

with $z_{i}=\left(x_{i}-q\left(E^{*}\right)\right) / \sigma_{q}\left(E^{*}\right)$ for $i=1,2$, where $x_{i}$ are the extrema of the interval

$$
\left[x_{1}, x_{2}\right]=[0,1.3] \cap\left[q\left(E^{*}\right)-3 \sigma_{q}\left(E^{*}\right), q\left(E^{*}\right)+3 \sigma_{q}\left(E^{*}\right)\right],
$$

and $\sigma_{q}\left(E^{*}\right)$ is the standard deviation of $q$, defined by

$$
\sigma_{q}\left(E^{*}\right)=\sqrt{\Gamma_{q\left(E^{*}\right)}}, \quad \Gamma_{q}\left(E^{*}\right)=\left[\frac{\partial q}{\partial E}\left(E^{*}\right)\right] \Gamma_{E}\left[\frac{\partial q}{\partial E}\left(E^{*}\right)\right]^{t} .
$$

\subsection{Probability of being a PHA}

We describe the computation of the probability that a local minimum value $d_{h}$ of $d$ is less than 0.05 AU. We stress that the use of the regularized minimal distances $\tilde{d}_{h}$ is essential for this purpose: for small nominal values of $d_{h}$ also negative values of the distance have to be admissible to perform the computation and the function measuring the minimal distance has to be regular also when it vanishes. The regularized map $\tilde{d}_{h}$ fulfills these properties and can be regarded as a random variable.

The probability that $\tilde{d}_{h}$ belongs to $[-0.05 \mathrm{AU}, 0.05 \mathrm{AU}]$ is

$$
\mathcal{P}\left(\left|\tilde{d}_{h}\right| \leqslant 0.05 \mathrm{AU}\right)=\frac{1}{\sqrt{2 \pi}} \int_{z_{1}}^{z_{2}} \exp \left(-z^{2} / 2\right) d z
$$

with $z_{i}=\left(x_{i}-\tilde{d}_{h}\left(E^{*}\right)\right) / \sigma_{\tilde{d}_{h}}\left(E^{*}\right)$ for $i=1,2$, where $x_{i}$ are the extrema of the interval

$$
\left[x_{1}, x_{2}\right]=[-0.05,0.05] \cap\left[\tilde{d}_{h}\left(E^{*}\right)-3 \sigma_{\tilde{d}_{h}}\left(E^{*}\right), \tilde{d}_{h}\left(E^{*}\right)+3 \sigma_{\tilde{d}_{h}}\left(E^{*}\right)\right],
$$

and $\sigma_{\tilde{d}_{h}}\left(E^{*}\right)$ is the standard deviation of $\tilde{d}_{h}$, defined by

$$
\sigma_{\tilde{d}_{h}}\left(E^{*}\right)=\sqrt{\Gamma_{\tilde{d}_{h}\left(E^{*}\right)}}, \quad \Gamma_{\tilde{d}_{h}}\left(E^{*}\right)=\left[\frac{\partial \tilde{d}_{h}}{\partial E}\left(E^{*}\right)\right] \Gamma_{E}\left[\frac{\partial \tilde{d}_{h}}{\partial E}\left(E^{*}\right)\right]^{t} .
$$

\subsection{Results of the computation}

In this section we show first the asteroids that are not present in the "official" list of NEAs given by the Minor Planet Center (MPC) $\dagger$, updated to August 4, and indeed have a non-negligible probability of being NEA or even PHA, we display the probability that they belong to such classes. Then we show the asteroids in the "official" list of NEA, that are not PHAs according to their nominal orbit, but are VPHAs.

The "official" lists of NEA and PHA are compiled by the MPC by using the elements of the nominal least squares orbits computed by the MPC. We recompute the least squares orbit, obtaining a slightly different result because of some different orbit determination algorithms and because we use of a different observations error model (see Carpino et al. 2003). Then we compute the uncertainty of both the perihelion $q$ and the (regularized) MOID. In Tables 3 to 8 we show the VNEAs and VPHAs that are not present in the list given by the MPC. These asteroids are divided into classes according to their Arc-Type (see Milani et al. 2006).

$\dagger$ It is maintained at http://cfa-www.harvard.edu/iau/NEO/TheNEOPage.html 


\begin{tabular}{lccc} 
VNEA & q & RMS & prob \\
\hline $1994 \mathrm{RD}$ & 0.4955 & 0.00122 & $100 \%$ \\
$2002 \mathrm{ON}_{4}$ & 1.2989 & $4.18 \times 10^{-6}$ & $100 \%$ \\
$2002 \mathrm{PT}_{140}$ & 1.2936 & $9.10 \times 10^{-6}$ & $100 \%$ \\
$2003 \mathrm{UW}_{26}$ & 1.2959 & $1.29 \times 10^{-5}$ & $100 \%$ \\
$2004 \mathrm{SB}$ & 1.3028 & 0.00359 & $22 \%$ \\
$1999 \mathrm{JS}_{6}$ & 1.3176 & 0.00831 & $2 \%$
\end{tabular}

Table 3. VNEAs not present in the list of NEAs given by the MPC: Arc-Type $\geqslant 5$

\begin{tabular}{lcccc} 
VPHA & dist & RMS & H & prob \\
\hline 1994 RD & -0.020 & $1.72 \times 10^{-4}$ & 17.21 & $100 \%$ \\
1994 RD & -0.034 & $1.61 \times 10^{-4}$ & 17.21 & $100 \%$
\end{tabular}

Table 4. VPHAs not present in the list of NEAs given by the MPC: Arc-Type $\geqslant 5$

First we take into account the best determined orbits, i.e. Arc-Type $\geqslant 5$. In Table 3 there are asteroids with a nominal perihelion distance very close to the boundary of the NEA class (i.e. 1.3 AU). This is a consequence of the use of an arbitrary boundary, not corresponding to a gap in the population: comparatively small differences in the nominal orbits computed by us and by the MPC result in a different classification.

We also find a very peculiar case: asteroid $1994 \mathrm{RD}$ is present in the table as a NEA with probability $100 \%$ and a nominal value of $q<0.5 \mathrm{AU}$; moreover it is present in Table 4 as a PHA at both local minima of $d$ with probability $100 \%$. Further investigations led us to the following conclusion: indeed $1994 \mathrm{RD}$ has been identified with $1991 \mathrm{AQ}$, and later numbered as (85182). It is present in the NEODyS website as (85182) 1991 AQ. Then why the name $1994 \mathrm{RD}$ is present in the database provided by the MPC? The answer is that a sort of administrative error occured: the arc of $1994 \mathrm{RD}$, made by observations done on September 2,4,5,6,7,11 1994, is not present in the observation file for (85182) which contains the observations of September 8,17,18,22,17,19 and October 3,4, 1994.

\begin{tabular}{lccc} 
VNEA & $\mathrm{q}$ & RMS & prob \\
\hline $2000 \mathrm{GM}_{146}$ & 1.044 & 0.020 & $100 \%$ \\
$2005 \mathrm{QN}_{87}$ & 1.281 & 0.048 & $65 \%$ \\
$2006 \mathrm{DV}_{62}$ & 1.230 & 0.001 & $59 \%$ \\
$2004 \mathrm{AT}_{1}$ & 1.503 & 0.564 & $36 \%$ \\
$2004 \mathrm{BD}_{11}$ & 1.305 & 0.012 & $35 \%$ \\
$2003 \mathrm{FF}_{42}$ & 1.701 & 1.306 & $28 \%$ \\
$2005 \mathrm{JK}_{173}$ & 1.647 & 2.079 & $22 \%$ \\
$2004 \mathrm{VT}_{16}$ & 1.315 & 0.017 & $18 \%$ \\
$2002 \mathrm{VF}_{118}$ & 1.478 & 0.178 & $16 \%$
\end{tabular}

Table 5. VNEAs not present in the list of NEAs given by the MPC: Arc-Type $=4$. We list only the asteroids with probability of being a NEA $>10 \%$.

The results for orbits with Arc-Type 4 are described in Table 5. The asteroid 2000 $\mathrm{GM}_{146}$ has probability $100 \%$ of being a NEA with nominal $q<1.05$ AU. This asteroid has been observed for 2 nights only, and it has not even an orbit computed by the MPC. We have found some VPHAs with Arc-Type 4 (see Table 6), but the probabilities are low.

In Tables 7,8 we show our results for less well determined orbits: these have ArcType $=3$, but are still considered reliable enough for claiming discovery of the corresponding asteroids, according to (Milani et al. 2006). 


\begin{tabular}{lcccc} 
VPHA & dist & RMS & H & prob \\
\hline $2004 \mathrm{AT}_{1}$ & -0.654 & 1.146 & 16.34 & $3 \%$ \\
$2003 \mathrm{FF}_{42}$ & -0.714 & 1.308 & 15.51 & $3 \%$ \\
$2005 \mathrm{JK}_{173}$ & -0.812 & 1.820 & 15.04 & $2 \%$ \\
$2002 \mathrm{VF}_{118}$ & -0.634 & 0.278 & 17.06 & $1 \%$ \\
$2004 \mathrm{BS}_{159}$ & -1.560 & 0.732 & 17.06 & $1 \%$
\end{tabular}

Table 6. VPHAs not present in the list of NEAs given by the MPC: Arc-Type $=4$.

\begin{tabular}{lccc} 
VNEA & $\mathrm{q}$ & RMS & prob \\
\hline $2005 \mathrm{UO}_{497}$ & 1.044 & 0.084 & $100 \%$ \\
$2005 \mathrm{TQ}_{79}$ & 1.112 & 0.034 & $100 \%$ \\
$2002 \mathrm{TO}_{301}$ & 0.947 & 0.040 & $100 \%$ \\
$2001 \mathrm{FN}_{91}$ & 0.974 & 0.007 & $100 \%$ \\
$2000 \mathrm{TF}_{2}$ & 1.289 & 0.007 & $94 \%$ \\
$2002 \mathrm{EP}_{150}$ & 0.928 & 0.254 & $93 \%$ \\
$2004 \mathrm{XZ}_{44}$ & 1.044 & 0.216 & $88 \%$ \\
$2005 \mathrm{PX}_{21}$ & 1.072 & 0.215 & $85 \%$ \\
$2005 \mathrm{JC}_{78}$ & 0.959 & 0.394 & $80 \%$ \\
$2005 \mathrm{YX}_{231}$ & 1.187 & 0.183 & $73 \%$ \\
$2001 \mathrm{SG}_{340}$ & 1.233 & 0.202 & $63 \%$ \\
$1999 \mathrm{CG}_{130}$ & 0.844 & 0.704 & $63 \%$ \\
$2003 \mathrm{LD}_{3}$ & 1.053 & 0.618 & $61 \%$
\end{tabular}

Table 7. VNEAs not present in the list of NEAs given by the MPC: Arc-Type $=3$. We list only the asteroids with probability of being a NEA $>60 \%$.

Note that asteroid $2005 \mathrm{UO}_{497}$, present as a sure VNEA and as a VPHA with $37 \%$ probability, has a constrained orbit (see Milani et al. 2005). Another interesting case is $2001 \mathrm{FN}_{91}$, that is both a VNEA and a VPHA with probability $100 \%$. The cases with the highest probability of being a PHA, namely $2001 \mathrm{FN}_{91}, 1999 \mathrm{CG}_{130}$ and $2005 \mathrm{UO}_{497}$, do not have an orbit computed by the MPC. This indicates that their orbit determination with the available data is not a trivial task. For $1999 \mathrm{CG}_{130}$ there are no photometric data, leaving doubts on the nature of the object; note that in this case the minimum distance could be less than 0.05 AU near both nodes.

\begin{tabular}{lcccc} 
VPHA & dist & RMS & H & prob \\
\hline $2001 \mathrm{FN}_{91}$ & -0.025 & 0.001 & 21.39 & $100 \%$ \\
$1999 \mathrm{CG}_{130}$ & 0.050 & 0.037 & - & $50 \%$ \\
$1999 \mathrm{CG}_{130}$ & -0.016 & 0.077 & - & $48 \%$ \\
$2005 \mathrm{UO}_{497}$ & -0.062 & 0.076 & 20.28 & $37 \%$ \\
$2005 \mathrm{JC}_{78}$ & -0.084 & 0.134 & 17.52 & $24 \%$ \\
$2005 \mathrm{PX}_{21}$ & 0.101 & 0.176 & 17.02 & $19 \%$ \\
$2002 \mathrm{EP}_{150}$ & 0.133 & 0.098 & 17.85 & $17 \%$ \\
$2004 \mathrm{XZ}_{44}$ & 0.089 & 0.284 & 19.01 & $13 \%$ \\
$2006 \mathrm{DA}_{72}$ & -0.067 & 0.311 & 15.67 & $13 \%$ \\
$2005 \mathrm{YX}_{231}$ & -0.197 & 0.184 & 19.52 & $12 \%$ \\
$2006 \mathrm{DZ}_{65}$ & 0.088 & 0.353 & 18.70 & $11 \%$ \\
$2005 \mathrm{RE}_{26}$ & -0.134 & 0.067 & 13.29 & $10 \%$
\end{tabular}

Table 8. VPHAs not present in the list of NEAs given by the MPC: Arc-Type $=3$. We list only the asteroids with probability of being a NEA $>10 \%$.

In Table 9 we display the VPHAs, found in the MPC list of NEAs updated to August 4, 2006, which are not PHA according to their nominal orbit. 


\begin{tabular}{lcccc} 
VPHA & dist & RMS & H & prob \\
\hline $1994 \mathrm{XG}$ & 0.063 & 0.030 & 18.58 & $33 \%$ \\
$2006 \mathrm{FW}_{33}$ & 0.066 & 0.111 & 20.12 & $30 \%$ \\
$2000 \mathrm{VZ}_{44}$ & -0.052 & 0.003 & 21.03 & $25 \%$ \\
$2006 \mathrm{FW}_{33}$ & 0.108 & 0.115 & 20.12 & $22 \%$ \\
$2006 \mathrm{KT}_{67}$ & 0.111 & 0.145 & 19.59 & $20 \%$ \\
$2006 \mathrm{CD}$ & -0.142 & 0.155 & 20.46 & $17 \%$ \\
$1999 \mathrm{UZ}_{5}$ & 0.055 & 0.004 & 21.87 & $12 \%$ \\
$1984 \mathrm{QY}_{1}$ & 0.179 & 0.084 & 14.16 & $6 \%$ \\
$2006 \mathrm{OV}_{5}$ & 0.192 & 0.090 & 19.02 & $6 \%$ \\
$2000 \mathrm{RK}_{12}$ & 0.056 & 0.004 & 21.27 & $5 \%$
\end{tabular}

Table 9. VPHAs (down to probability $>5 \%$ ) in the "official" list of NEAs, that are not PHAs according to their nominal orbit.

\section{Conclusions and future work}

We have done some progress in the understanding of the mutual geometry of two confocal Keplerian orbits: this seems to be a fairly difficult problem and there are still some interesting geometric features to investigate. The regularization of $d_{h}$ and $d_{\text {min }}$ explained above allows to define a meaningful uncertainty of the local minima of $d$ even if this uncertainty leads to negative values of the distance. Moreover the orbit crossing singularity is removed, except for the tangent crossing case. We have computed the uncertainty of the local minima of $d$ for several thousands of asteroids, whose orbit uncertainty is not too large, and for all the known NEAs. By this computation we have found some VPHAs among asteroids that are not even NEAs, according to their nominal orbits. The effect of the nonlinear terms, neglected in the determination of the orbit uncertainty and in the covariance propagation formula, still needs to be investigated. We expect that if the orbit of an asteroid is poorly determined, like for objects whose observations form an arc with Arc-Type 2, then the uncertainty of $\tilde{d}_{h}$ and $\tilde{d}_{\text {min }}$ might need to be computed with a method taking properly into account the nonlinearity of the orbit determination. This is the target for our future work.

\section{References}

Bini, D. A. 1997, Numer. Algorithms 13, 179

Bonanno, C. 2000, Astron. Astrophys. 360, 416

Bowell, E. \& Muinonen, K., 1994, in: T. Gehrels (ed.), Hazards due to comets $\&$ asteroids, (Tucson: The University of Arizona Press), p. 149

Carpino, M., Milani, A. \& Chesley, S. R. 2003, Icarus 166, 248

Cox, D., Little, J. \& O'Shea, D. 1992, Ideals, Varieties and Algorithms, Springer-Verlag

Dybczynski, P. A., Jopek, T. J. \& Serafin, R. A. 1986, Celest. Mech. \& Dynam. Astron. 38, 345

Gronchi, G. F. 2002, SIAM Journ. Sci. Comp. 24, 61

Gronchi, G. F. 2005, Celest. Mech. E Dynam. Ast. 93, 297

Gronchi, G. F. \& Tommei, G. 2007, $D C D S-B, 7,755$

Hoots, F. R. 1984, Celest. Mech. E Dynam. Astron. 33, 143

Jazwinski, A. H. 1970, Stochastic processes and filtering theory, Academic Press

Kholshevnikov, K. V. \& Vassiliev, N. 1999, Celest. Mech. \& Dynam. Astron. 75, 75

Milani, A. 1999, Icarus 137, 269

Milani, A., Sansaturio, M. E., Tommei, G., Arratia, O. \& Chesley, S. R. 2005, Astron. Astrophys. 431,729

Milani, A., Gronchi, G. F. \& Knežević, Z. 2006, Earth, Moon 8 Planets, in press

Milnor, J. 1963, Morse theory, Princeton University Press

Sitarski, G. 1968, Acta Astron. 18, 171

Wetherill, G. W. 1967, J. Geophys. Res. 72, 2429 